\title{
A Novel Routing Algorithm in Wireless Ad Hoc Network
}

\author{
Zuchun Ding ${ }^{1}$, Wenying $\mathrm{Mo}^{1}$, Jian Chen ${ }^{2}$, Lei Lei ${ }^{3}$, Xinghong Qin ${ }^{4}$ \\ 1. Guangdong AIB College, Guangzhou, China \\ 2. Guangdong Zhongya Wireless Tech. Co. Ltd \\ 3. Guangzhou Communication Institute, Guangzhou, China \\ 4. Beihang University, Beijing, China
}

zucding@gmail.com, mowenying@126.com, 13928788668@139.com, leileicr@gmail.com, billqin@163.com

\begin{abstract}
This paper suggests a novel algorithm of wireless ad hoc network. This algorithm has the features of adaptive clustering, multiple layering and treelike topology, with excellent abilities in autonomous network construction. The algorithm also suggests effective measures for handover and communication in position changing. This routing algorithm can construct robust network, because of the changeable of every nodes. Every node in this algorithm has the function of gateway and can connect two subnets together. In practice, the algorithm has good abilities in emergency communication and military environment where infrastructure networks are often inaccessible.
\end{abstract}

Keywords- Wireless, Ad hoc, Network; Route, Adaptive Clusterin

\section{INTRODUCTION}

In mobile communication, wireless ad hoc network has the features of strong mobility, without the need of infrastructures, and attracts more and more attentions. In this region, the routing algorithm is the key technology, which determines the network structure, communication specifications and network robustness. But the authentication anti-ruin ability, communication loads, speed etc. problem aren't yet solved effectively [1-6].

In this paper, a novel routing algorithm is supposed and it has been used in practicable wireless network construction, and the problems introduced above are solved effectively. It's comprised by network topology and routing procedure introduced below.

\section{Multilayer TreeliKe Topology AND AdAPTIVE CLUSTERING}

In ad hoc network, the flexible routing algorithm is the key factor to strengthen the mobility of networks. To get high capability of network, we consider in three factors, i.e. layering, treelike topology and adaptive clustering. This algorithm has been applied to practical work, namely wireless autonomous ad hoc routing networks.

\section{A. Adaptive clustering}

Firstly, we consider the multiple mobile communication nodes distributed in a wide region. Every node gives out hello data package with the node identification information such as node number, battery capability ad so on. This procedure will produce every cluster and vote the cluster head of each cluster. Only the node in the most center position and with more power capability and some other virtues has the chance to be voted as cluster head. In practice, considering the important factors, we use the weighting sum of several variables, i.e. the position in a cluster, power capability, propagating loss, and so on, the sum is produced by multiplying the weights with these factors, and the node with the biggest sum is voted as the cluster head.

We select the factors considered in the sum introduced above for the reason that the node in the center position will emit signals in less power, because the node as cluster head can communicate with other nodes in this region with lower power, and the power capability will determine whether the node can work continually without the lacking of power supply. For its importance, 0.3 is selected as the coefficient or weight of this factor. When the propagation loss is high, the signal received will have low SNR. The propagation loss is determined mainly by reliefs, plants and buildings in the surface of earth, and the distance between the source node and target node. So these factors are important when cluster heads are selected.

This process is illustrated in Figure 1.

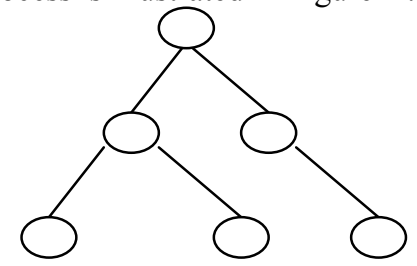

Figure 1. Treelike routing cluster structure

Here, every circle is a node of wireless ad hoc network. The lines between nodes are the routes between nodes. The root node is also the head of this cluster, and is also a gateway when the next hop is beyond the same kind network and with different protocols.

The network structure should suit for not only little scale network, but also for large scale network with much nodes. If single treelike structure is used, it will result in too deep in network depth, and transfer delay will be long, and the more near the root node, the data flux will be higher. In some circumstances, the route is even obstructed. If the root node is in trouble or ruined, the all network will fail. So the depth 
of a treelike topology should be appropriate, in practice less than 4.

The treelike structure is the elementary unit of a wireless ad hoc network. These units comprise an ad hoc network, as illustrated in Figure 2.

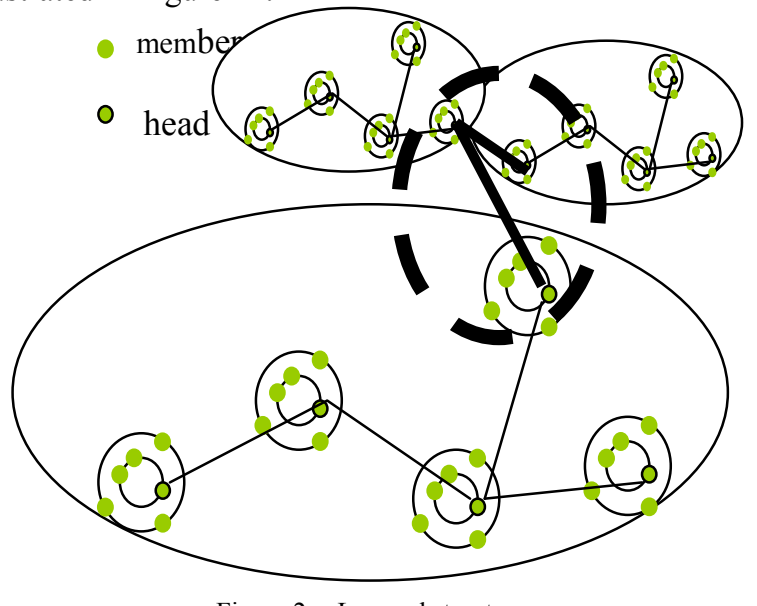

Figure 2. Layered structure

In this figure, every concentric circle represents an Ad hoc network unit, i.e. cluster illustrated in figure 1.

The nodes in different clusters communicate via cluster heads. Cluster heads in the same region illustrated by one ellipse form a treelike cluster in an upper layer.

By the same reason, the nodes in upper layer form the third layer treelike clusters. In this figure, an example is illustrated in the circle with bold line.

This structure is convenient to communicate between nodes, and is also in accordance with the managing structure in university, corporation and so on, and therefore is practicable.

\section{B. Multi-layer structure}

In practice, the management in any university or corporation is executed layer by layer. In fact, the networks discussed here are also designed layer by layer.

When the preliminary clusters are formed, every node is a member of the cluster which it belonged to. Members in the same cluster communicate with each other in the region of this cluster. Every cluster votes a head and the heads of all clusters form clusters in the second layer, and the heads of clusters are also voted. The rule of cluster forming and head voting in the upper layer is the same as the rule of first layer. In the first layer, members in different clusters communicate via cluster heads of the cluster which the source node or the target node belonged to. When necessary, the third layer can be formed. In this structure, every node in the upper layer is the cluster head of the lower layer. Every cluster head communicates with cluster members and other cluster heads in different frequencies to avoid interferences between each other.

\section{Treelike routing structure}

Every cluster is formed in treelike structure. The reason of this structure is that the data flow in this structure is lower than other structures, and the routes in this structure are moderate than those of mesh structure.

The treelike routing structure is illustrated in figure 1. Every segments between nodes is a possible route.

\section{CORE DATA STRUCTURE}

There are many data structures for ad hoc network routing technology in our algorithm. Three basic data structures are core structures, i.e. host table, neighbor table and route table.

\section{A. Route table}

This table is the collections of all hosts. The data structure is listed below.

TABLE I. Host TABle Structure

\begin{tabular}{|l|l|}
\hline Data item & Data type \\
\hline Host name & char \\
\hline Id information & number \\
\hline Belonging to & char \\
\hline others & \\
\hline
\end{tabular}

In multi-layer structure, the nodes in every cluster have the different host table, but in the same layer, the host table in every node is the same. The host names in upper layer are the collections of the cluster heads of lower layer.

\section{B. Neighbor table}

This table is the collections of all neighbor nodes. The data structure is listed below.

TABLE II. NeIGHBOR TABLE STRUCTURE

\begin{tabular}{|l|l|}
\hline Data item & Data type \\
\hline Host name & char \\
\hline Neighbor host & char \\
\hline Signal strength & number \\
\hline others & \\
\hline
\end{tabular}

Here, the item Signal strength indicates the quality of the signals received, and it's used to calculate the wave propagation loss. It's useful when cluster heads are voted.

\section{Route table}

This table is the collections of all routes. The data structure is listed below.

\section{TABLE III. ROUTE TABLE STRUCTURE}

\begin{tabular}{|l|l|}
\hline Data item & Data type \\
\hline Target host address & char \\
\hline Source host address & char \\
\hline Hops & number \\
\hline Next hop node & char \\
\hline Changing ID & char \\
\hline Pre-node & char \\
\hline others & \\
\hline
\end{tabular}

Router table comprises the selected route and prepared route, these data are extracted from route table and neighbor table. Pre-node in the table is the starting node in a route. 


\section{Priority table}

In our routing algorithm, a priority table is necessary. See table IV.

This table can supply Quality of Service (QoS) management function in network communication.

TABLE IV. PRIORITY TABLE STRUCtURE

\begin{tabular}{|c|l|}
\hline Priority rank & Description \\
\hline 7 & Out-net control \\
\hline 6 & In-net control \\
\hline 5 & Urgent order \\
\hline 4 & Extreme rapid \\
\hline 3 & Rapid \\
\hline 2 & At once \\
\hline 1 & Considered privilege \\
\hline 0 & No privilege \\
\hline
\end{tabular}

\section{ROUTING PROCEDURE}

\section{A. Distributed authentication}

To get a robust and rapid authentication, distributed authentication is needed. Traditionally, concentrated authentication is often used in wireless network, i.e. a specified server is used as authentication center. But concentrated authentication has the fatal shortcoming that the network is thereby becoming vulnerable and even ruined. If the authentication sever is ruined, then the whole network will become paralyzed, because all the identification information is stored in this server only and all decisions about nodes are thereby determined by the unique sever.

In the common senses, we know one can identify other people by the information known by himself, unnecessary to get information from a specified person like an authentication server, unless his knowledge is not enough. In this kind of identification, authentication is faster than authenticated by a specified person. In fact, this kind of authentication is distributed, and has the virtues of rapidness, anti-ruined ability and robustness.

Here, the routing strategy uses this kind of distributed authentication method suggested above. By this method, authentication anti-ruin ability is improved.

\section{B. Wireless network topology formation}

In the beginning, it's supposed that every node knows nothing about his neighbor nodes. Nodes must exchange their topology information with each other to construct entire network topology.

\section{Basic treelike routing topology formation}

Nodes construct basic routing tree, and the node in the root is the cluster head, the neighbors are leaves. The amount of leaves in a tree is limited to 1 to 8 for practice to avoid over expended network. Certainly, the root can communicate with its leaves directly or via only several hops.

Every basic routing tree exchanges routing information after routing tree is formed to store the whole routes.

\section{Exchanging routing information}

Every node broadcasts its own routing tree information. After the adjusting of topology information, the entire topology is formed.

For example, nodes A and D have the treelike topology shown by Figure 3 and Figure 4 .

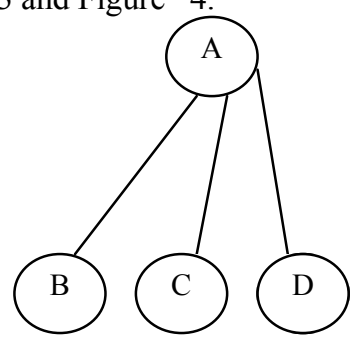

Figure 3. Tree structure with root node A

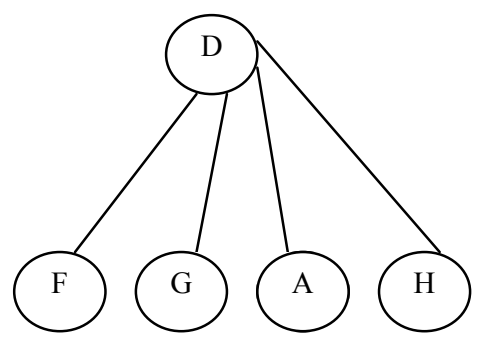

Figure 4. Tree structure with root node D

Node A gets the routing tree information of node D after routing tree information exchanging. Then node A link node $\mathrm{D}$ into the tree that $\mathrm{A}$ belonged to, and $\mathrm{A}$ get the routing structure shown by Figure 5 .

In the procedure of linking routing table, redundant routes are deleted, and eventually an entire route table is formed. In practice, the node letters are substituted by IP addresses.

By means of this method, an entire network topology is get eventually.

Because entire information exchanging will exhaust large amount of network resources, for a fully connected network, the cost is proportional to the square of node amount, therefore the data without too much information should be deleted.

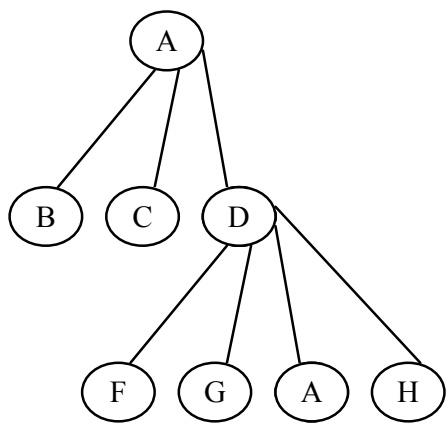

Figure 5. Linking the node $\mathrm{D}$ into the tree node $\mathrm{A}$ belonged to 
For this reason, routing tree information is processed by the rule as followed.

Only the shortest route is remained from root node to another node;

If more than two routes are with the same length, then two routes are all remained, in which a extra route is prepared as redundant route;

When nodes detect the changing of topology, the changing information will be used to consider whether the topology of the local network should be adjusted or not.

\section{E. Resources-economic information transfer}

In this algorithm, the routing information is conveyed only between the minimum sub-tree before and after the node moves. The network resource is thereby saved because the decreased amount of broadcast nodes is limited.

In the view of the routing procedure, this algorithm restricts the unnecessary routing information. In tradition, neighbors in an ad hoc network exchange large amount of routing information frequently, so that many broadcasting data are produced. In this algorithm suggested here, routing information are conveyed from son-node to father-node, and only when the location is changed or new nodes are connected, therefore the data conveyed are much lower.

In the data conveying procedure, the data packages access the convergence node of a sub-tree by default route, then convey to target node from this convergence node hop by hop.

Routing protocol uses the virtues of the routing on demands protocol and active routing protocol. Every node consists of the routing information to other nodes. The node information will be refreshed periodically. This algorithm can support the minimum communication delay. The routing sequences have the virtues to prevent the circulation in routing.

By this method, communication loads are little, and communication speed is high.

\section{EXPERIMENT RESULT}

Using the algorithm above in network designing, we get the practiced work result as followed. Here, the data structures listed in part III are used.

The performances we get are listed below.

Maximum hops $>16$;

Supporting TCP/IP, clustering sub-tree and multi-layered structure;

Convergence time increases less than 2 seconds when a hop is added;

Dynamic topology, nodes can be connected or quit;

Distributed authenticating rapidly;

Continuous communicating when changing node location; Node amount in the network $>1000$.

\section{CONCLUSIONS}

In this paper, we make an extensive research on wireless ad hoc networks, propose a tree-like multilayer adaptive clustering routing algorithm, the work result prove the effectiveness of this method. This method solves the problem of authentication anti-ruin ability, communication loads, speed effectively in practice.

\section{REFERENCES}

[1] Charles E. Perkins, "Ad hoc Networking", Addison-Welsley, Dec 2000 .

[2] Yingji Zhong, "Dynamic source routing protocol for wireless ad hoc networks in special scenario using local information", Communication Technology Proceedings, ICCT 2003. International Conference, Vol.2, April 9-11, 2003, pp. 1287-1290.

[3] Johnson D B., "Dynamic Source Routing in Ad Hoc Wireless Networks", Mobile Computing, Ch5, 1996, pp.153-181.

[4] Zhou A., "Load balanced wireless ad hoc routing, Canadian Conference on Electrical and Computer Engineering" , Vol. 2,May 2001, pp. 1157-1161.

[5] Yuan Xue; Baochun Li; Klara Nahrstedt, "Optimal resource allocation in wireless ad hoc networks: a price-based approach", IEEE Transactions on Mobile Computing, April 2006 , pp. 347-364.

[6] Biagioni, E.S.,"Algorithms for Communication in Wireless multi-hop ad hoc Networks using Broadcasts in Opportunistic Large Arrays (OLA)", Proceedings of 16th International Conference on Computer Communications and Networks, 2007. ICCCN 2007, pp. 1111-1116. 